\section{Cultural diversity drives innovation: modeling in the global pharmaceutical industry}

\section{Cultural diversity drives innovation}

\author{
Graham Jones
}

TRD INNOVATION, Novartis Pharmaceuticals Corp, East Hanover, New Jersey, USA

Bernardita Chirino Chace

TRD INNOVATION, Novartis AG, Basel, Switzerland, and

Justin Wright

TRD INNOVATION, Novartis Pharmaceuticals Corp, East Hanover, New Jersey, USA

\begin{abstract}
Purpose - The innovative capacity of an organization is typically realized through unit-level teams. Previous studies correlate innovation performance with cultural diversity of teams, but note that team dynamics need to be optimized to derive maximum benefit. Herein, this study offers an assessment of available team building instruments through the lens of diverse innovation teams. In a demonstration project in the pharmaceutical industry, this study then outlines specific tools and approaches which can be successfully deployed through team coaching and mentoring.

Design/methodology/approach - A cluster of nine innovation teams with varying degrees of cultural diversity was provided with assessment and management instruments which had been identified and field tested by a mentoring team. Content included cultural awareness tools, innovation team profiling methods and Team Science (SciTS) ideology. Teams were funded, coached and mentored through a six-month performance period and assessed at regular intervals.

Findings - Team assessments provided correlations between performance (measured by project completion and new intellectual property generated) and diversity together with wealth of information on intra-team culture and dynamics. Concrete recommendations from the study include adoption of appropriate communication standards to promote inclusivity, use of SciTS operational tracking metrics to enhance engagement, use of the FourSight group profiling methodology and cultural quotient scale cultural awareness instruments at team-forming stage to promote effective dynamics and enhance inclusivity.

Practical implications - Cultural diversity has a positive impact on innovation teams. This said, for maximum benefit cultural awareness of team members should be optimized to avoid unintended conflicts developing. Such issues can be exacerbated when teams are deployed remotely and preventative measures should be established. These issues became of heightened significance as a result of telecommuting imposed by the COVID-19 pandemic and have longer-term implications, as corporations consider global air travel reduction through environmental concerns. A tracking tool is described to monitor team engagement and promote inclusivity. It is expected that the learnings can influence how teams can best form, normalize and operate within corporate innovation programs and form the basis of long-term impact studies.
\end{abstract}

(C) Graham Jones, Bernardita Chirino Chace and Justin Wright. Published by Emerald Publishing Limited. This article is published under the Creative Commons Attribution (CC BY 4.0) licence. Anyone may reproduce, distribute, translate and create derivative works of this article (for both commercial \& non-commercial purposes), subject to full attribution to the original publication and authors. The full terms of this licence may be seen at http://creativecommons.org/licences/by/4.0/legalcode

The authors wish to thank Elena Rodriguez, Anastacia Awad, Ivonna Demme, Nancy Long, Christian Pihlgren, Unmesh Deodhar, Rahul Sharma and Clara Fernandez de Castro for inputs on the manuscript.

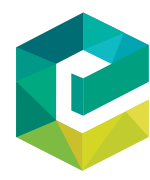

International Journal of Innovation Science

Vol. 13 No. 2, 2021 pp. $133-144$ Emerald Publishing Limited 1757-2223 
IJIS

13,2

Originality/value - This represents the first systematic study on the impact of cultural diversity and team dynamics within innovation programs in the pharmaceutical industry. The tools and methodologies deployed are widely available and can be adopted by innovation teams in many adjacent industries with established innovation ecosystems.

Keywords Implementation, Assessment, Innovation, Culture, Diversity, Inclusion, Ideation, Team science

Paper type Case study

\section{Introduction}

Diversity and inclusion are cornerstones of modern day industry and strategies to enhance, expand and champion have reaped dividends in all sectors (De Abreu Dos Reis et al., 2007). In addition to helping establish inclusive environments which model and represent society at large there is growing recognition that this provides competitive commercial advantage (Lorenzo et al., 2018; Jones et al., 2020). Over millennia, our evolutionary path has relied on genetic diversity to drive growth through provision of competitive advantages in the face of new environmental challenges. It is not hard to extrapolate this in the business world, where market pressures and competition can render successful organizations obsolete within a matter of years, making adaptive change and innovation sine qua non. In the scientific and technical world, there is evidence that diverse teams produce outputs with greater value, and mounting interest on the impact of diversity on innovation processes, where lateral thinking at ideation phase, is assumed to be at a premium (Shane, 1993). As a global pharmaceutical company, Novartis has made great strides increasing diversity at boardroom level, senior leadership and middle management, making it one of the preferred destinations in the industry. Buoyed its vision of "reimagining medicine", innovation has become a central tenet of strategy and growth across the entire organization (Novartis, 2020). Mounting evidence suggests that in the case of innovation activities, cultural diversity of teams has a significant beneficial impact on performance (Bertelsmann, 2018) and a direct correlation with increases in innovation revenues (Lorenzo et al., 2018). The focus of the work herein is a case study conducted in one of our primary organizational units, Technical Research and Development (TRD). For the purposes of this manuscript the term innovation refers to the processes of ideation, design, initiation and subsequent implementation of novel scientific approaches and principles for the development of new products.

\section{Technical Research and Development innovation program: catalyzing cross- functional ideation}

As a global organization, Novartis has 109,000 employees representing over 140 nationalities located across six continents in over 30 countries. Within the company, the TRD division represents the Chemistry and Manufacturing Controls arm of the corporation and has 2,900 employees distributed over 6 sites in Europe, 4 in Asia and 3 in North America. To bolster innovation activities throughout the ecosystem, TRD established an enterprise wide Innovation Program in 2018. Supported by a core management team, the functional activities of program are overseen by an appointed Innovation Council (IC). Within its first year of operation, the program launched numerous initiatives to promote awareness on innovation, including an education syllabus, a $24 \mathrm{~h}$ global innovation day, innovation project portfolio mapping, and a seed funding mechanism to allow teams to work on cross-functional high-risk high-reward research projects related to line function needs (Nelson, 1991). The IC is composed of three executive leads and ten appointees nominated from the various TRD line functions. Gender balance is male (8) to female (5) and members hail from a total of nine different nations of origin. Inspired by this level of representation, the IC made diversity and inclusion an area of 
concentration within its work streams, with a goal to study and develop best-in-practice tactics that can be deployed across the organization. The impacts of cultural origin on values and behavioral drivers have been studied in some depth by Hofstede and others (Hofstede, 2010; Hampden-Turner and Trompenaars, 2006). Mapped on a global scale against six metric "dimensions", the calibrated indices cover 110 nations and prompted research on relationship between nation of origin and innovation performance, which revealed some direct correlations (Kaasa and Vadi, 2010). Illustrative data for the IC membership across two relevant dimensions are presented in Figure 1, namely, the "power distance index" (PDI) (where a negative relationship with innovation has been suggested) and IDV or "individualism" (positive relationship suggested). Wide scoring ranges (0-100 scale) are typically observed in the indices across each of the six dimensions and although intra-country regional differences are masked through aggregation, the data has nonetheless proven useful as a reference model. Separate national-level studies have examined links between culture and innovation output with interesting findings which have been used to inform economic development initiatives (e.g. Dakhli and de Clercq, 2004).

In efforts to understand cultural and cross-cultural dynamics, social researchers have identified a total of ten major cultural clusters globally, one of which the majority of nations are identified with, namely, Anglo, Arabic, Confucian Asian, Eastern European, Germanic European, Latin American, Latin European, Nordic, South Asian and Sub-Saharan Africa (SHRM, 2015). Noteworthy for the TRD IC is that seven of these ten clusters are represented by its members. However, though cultural diversity on teams is considered to provide creative advantage (diversity in approach to analysis, problem solving, risk taking, etc.), it has been noted that high team diversity can also lead to conflict if not managed appropriately (Bertelsmann, 2018). Differing value systems, methods of communication and interpretation among team members have the potential to lead to mis-understanding and mis-conception if cross-cultural awareness is low. As a simple example communication style preferences range from direct (where the spoken word is taken literally) to indirect (where context is of high importance) and pronounced differences are observed across nations and

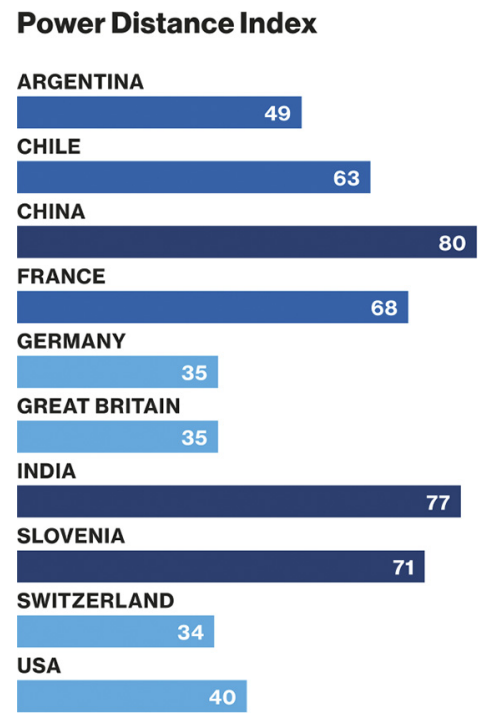

\section{Individualism v Collectivism}

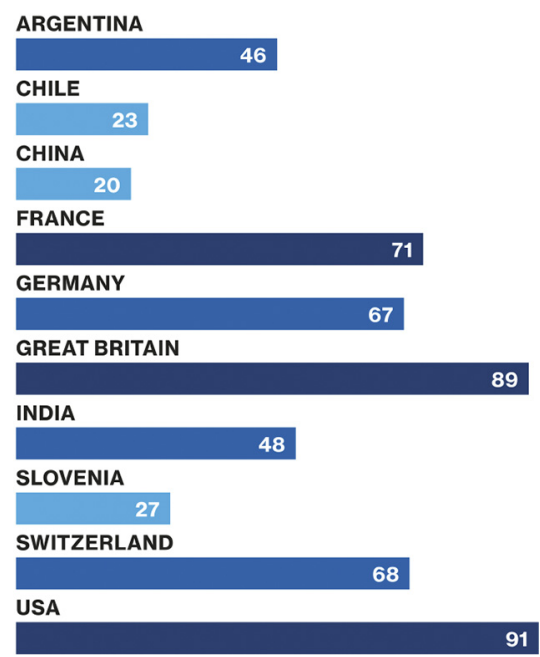

\author{
Cultural \\ diversity \\ drives \\ innovation
}

135 
cultures (Gudykunst and Ting-Toomey, 1988). Accordingly, it is important that cultural sensitivities are reflected in team operating principles in order to establish highly inclusive environments where the full benefits of team diversity can be exploited. The IC thus elected to evaluate various team strengthening and assessment instruments as part of their mentoring program for innovation teams. Several excellent customized programs were available in house and IC members recalled experiences with similar programs from previous employers. However, utility was also seen in evaluating commonly available platform tools and programs with a view to identifying content that could be deployed industry-wide. Over a series of offsite retreats, numerous methodologies were examined through the lens of supporting diverse and inclusive innovation teams (Matsumoto and Hwang, 2013; Nakata and Sivakumar, 1996). Of these, eight were selected for field testing and consideration for deployment. Metrics selected included cultural awareness, diversity and inclusion measures, psychological safety of members and ability to nurture ideation. Evaluation was performed through a combination of online testing followed by group discussion [Myers-Briggs type indicator, FourSight, Belbin, cultural quotient scale (CQS)], live team exercises (de Bono, Mayo Clinic CfI, Corporate Startup) and workshops (Team Science [SciTS]). As depicted in Table 1, the FourSight thinking preferences instrument were scored highly, as was the CQS assessment program for cultural awareness. Another stand out was the SciTS training toolkit which serves as key instrument for driving inclusivity within teams.

A composite of the FourSight preference profiles across the IC membership is depicted in Figure 2. Using this survey instrument, respondents are categorized based on high/low preferences across four thinking profiles (namely, ideator, implementer, developer and clarifier). A total of 15 unique profiles are in fact possible, stemming from single peak preferences (ID, IM, DV or CL) plus double, triple or even four way combinations thereof (Bratsberg, 2012). Perhaps unsurprisingly, Ideation (ID) and Implementation (IM) were high preferences for nearly all IC members, and there were also four "drivers" (double combinations) which have high preference for ID and IM as their basis. Absent among the group was the high developer DV preference which may reflect the IC's early stage ideation tendencies, but signals a clear need for the group to collaborate with DV and also CL preferring individuals (of which there were a total of four among the group) to support program refinement and development. Interestingly, no integrators (who have an even balance of all four preferences) were recorded within the group.

Members also participated in CQS assessment profiling which, based on a series of inter-related and weighted responses, generates a composite profile based on four attributes: the respondents cultural quotient (CQ) knowledge, CQ strategy, CQ action and CQ drive (Jones et al., 2020). The IC members generally scored highest in the CQ drive component (all scales 0-100 against mean for all respondents), possibly reflecting motivation toward the subject matter. The largest variation of scores was for the socio-linguistic component within CQ knowledge, reflecting the fact that many members are multi-lingual and some monoglot. Favorable assessment of the SciTS toolkit was driven by multiple components, including effective team forming guidelines, fault line awareness, dialog and debating schemas, inclusive operating principles and conflict resolution methods (Lau and Murnighan, 1998; Thomas and Kilmann, 1978). Inspired by learnings from the toolkit, a project/ team tracking instrument was developed for deployment with innovation teams (vide infra).

\section{Demonstration project: the X-functional innovation team innovation seed funding program}

One of the key work streams of the IC was to establish a funding mechanism wherein teams of aspiring innovators submit proposals in defined thematic areas of interest. A variety of funding 


\begin{tabular}{|c|c|c|c|c|c|}
\hline $\begin{array}{ll} & \text { Drivers } \\
\text { Instrument } & \end{array}$ & $\begin{array}{l}\text { Cultural } \\
\text { Awareness }\end{array}$ & $\begin{array}{l}\text { Inclusive } \\
\text { Behaviors }\end{array}$ & $\begin{array}{l}\text { Interpersonal } \\
\text { Interactions }\end{array}$ & $\begin{array}{l}\text { Classifiers \& } \\
\text { Identifiers }\end{array}$ & $\begin{array}{l}\text { Ideation } \\
\text { Culture }\end{array}$ \\
\hline $\begin{array}{l}\text { MBTI Assessment } \\
\text { (Higgs, 1996) }\end{array}$ & + & + & + & ++ & + \\
\hline $\begin{array}{l}\text { Four Sight Assessment } \\
\text { (Bratsberg, 2012) }\end{array}$ & + & ++ & +++ & ++ & +++ \\
\hline $\begin{array}{l}\text { Belbin Assessment } \\
\text { (Belbin, 2010) }\end{array}$ & + & ++ & ++ & ++ & + \\
\hline $\begin{array}{l}\text { de Bono's Hats Modeling } \\
\text { (Kivunja, 2015) }\end{array}$ & + & ++ & + & ++ & ++ \\
\hline $\begin{array}{l}\text { CQS Assessment } \\
\text { (SHRM, 2015) }\end{array}$ & +++ & +++ & ++ & ++ & + \\
\hline $\begin{array}{l}\text { Mayo Clinic Cfl Caricatures } \\
\text { (Van Wulfen, 2009) }\end{array}$ & + & + & ++ & ++ & +++ \\
\hline $\begin{array}{l}\text { Corporate Startup Characters } \\
\text { (Viki, 2016) }\end{array}$ & ++ & + & ++ & ++ & +++ \\
\hline $\begin{array}{l}\text { SciTS Toolkit } \\
(\mathrm{NIH}, 2010)\end{array}$ & ++ & +++ & +++ & + & + \\
\hline
\end{tabular}

Cultural diversity drives innovation

Figure 2.

Assessment and coaching instruments evaluated by the TRD innovation council

mechanisms are currently available through the functions via short, medium and longer term horizons typically tied to existing business objectives of the relevant organizational unit. However, we implemented an additional program (X-functional innovation team [XFIT] which was designed to attract new entrants to the ecosystem and provide the opportunity for white space innovation. A premium was placed on the cross-functionality of the team (reflective of the program XFIT) together with the originality of the proposed project. Following peer review, a total of 9 teams were selected from an applicant pool of $>200$, and each team was assigned a coach identified by the IC. A deliberately tight timeframe of 6 months support was given to each team, to promote "learn/fail fast" innovation culture. Coaches were serial innovators, with knowledge of SciTS concepts and methodology obtained through internal workshops and training. Each team was also provided the opportunity to complete the FourSight thinking preferences program and CQS assessment, with results made available to the individuals and composites provided to the team.

Teams traditionally form on the basis of common subject matter interest for any envisioned project. Unlike many interdisciplinary team projects in the academy (where a considerable proportion of research on team dynamics has been performed), in the global pharmaceutical industry, it is not uncommon to find teams composed of members located in several countries, and with a considerable breadth of nationalities. Given the purported advantages of cultural diversity for innovation, studying such teams presented an excellent opportunity to examine key components of team dynamics (Horwitz and Horwitz, 2007; Jackson et al., 1995). Potential touch points could include the impact if any of language barriers, cultural values, methods of interpretation, risk tolerance and communication styles (Moon, 2013; Amiri et al., 2010). For some teams, additional considerations may emerge from inherent geographic challenges and consideration of time zones and adapting to telecomsbased meetings (Koehne et al., 2012; Gibson and Gibbs, 2006). It was reasoned that learnings from such a study could help champion the impact of cultural diversity and best practice inclusive behaviors and prove useful for future teams, including those that were formed deliberately and not solely based on common subject matter interest of the members.

Most of the nine XFIT teams contained members originating from several countries, and many were located in different continents. Cultural clusters represented per team ranged from 7 (1 team), 5 (2 teams), 4 (1 team), 3 (2 teams) and 2 (1 team), with two teams 
representing a single cluster. Team gender balance generally reflected norms associated with the line functions involved in the projects. The coaches engaged in on-site and remote mentoring with specific check in intervals, and upfront training on financial aspects of project management was offered through workshops. Administrative coordination of the projects was conducted centrally (Basel, Switzerland) with high emphasis on budget tracking (funding from September 2019 to March 2020 crossed two fiscal years) and personnel deployment (interns, students). Toward the conclusion of the program, while the teams were still active, a multi-faceted questionnaire-based interview was completed by each team to assess specific criteria. Additionally, team coaches were interviewed, and each team presented results to the IC followed by Q\&A session.

\section{Team findings}

Generally, each project was initiated within one month of the funding start date, and regular updates were provided to the coaches. Generic problems encountered by the teams included delays in personnel recruitment and procurement tracking. Feedback solicited from the teams centered on project progress and ideation levels, and additional insights were probed around key drivers:

- team size;

- sub-group formation;

- time zone differences;

- hub and spoke relationship of the team;

- meeting modality;

- frequency of meetings;

- communications style; and

- perceived level of inclusivity within team.

Most teams met or exceeded original project goals and many resulted in generation of additional IP as a result of new avenues of investigation. Larger teams (range was $n=4$ to $n=13)$ reported that for effective management it required the formation of sub-teams $(\sim 5$ members) which either centered on scientific groupings and/or facilitated reasonable accommodations for time differences (US-European and European-Asian being the most common). The smaller sub-teams were also better prepared for rapid sprint cycles for ideation which benefit from regular and sustained group check-ins. The meeting frequency of full teams ranged from weekly to six weeks. Team leaders and members saw value in both the CQS and Four Sight assessment instruments, which elicited high engagement levels. Several members had already participated in internal trainings for team management and cultural awareness, including the "M1-neuro linguistic programming" program (Neuro Linguistic Programming) which has similarities to the Four Sight assessment. Leaders in particular saw value in improving effectiveness interacting with members with different value systems and thinking profiles, allowing them to disarm overly vocal members to promote inclusivity. Most agreed that pro-active steps need to be taken to help engage team members who have less assertive styles and the suggestion of having a member specifically act as an inclusivity observer was advanced. He/she would be responsible for monitoring verbal and non-verbal cues in the meeting (including online video feeds for remote participants) including inviting members to speak and diffusing potential conflict points. It was also suggested that in-room and remote participants could benefit from an electronic system to democratically queue questions from members in the order they were prompted, 
and the meeting chair would adhere to the system (modifications of existing meeting room apps/programs such as Skype, Microsoft Teams, etc. is being investigated). Improvement of general meeting etiquette to reflect cultural sensitivities was also suggested ranging from refraining from publically characterizing "good ideas" (which may, by default send the un/ intended and potentially demoralizing appearance of implying all other ideas pre/prior were lesser) to avoidance of pre-meeting "ice breaker" conversations which may have culturally exclusionary content (e.g. sporting events, political biases, etc.) in favor of open ended dialogs, e.g. "who did something interesting this weekend"? There was general agreement that for a more standardized and codified approach to team development, a tracking and evaluative tool would be of use, allowing all members to contribute feedback anonymously at specific time points in the projects. A prototype was developed (Figure 3) based on a combination of SciTS principles (Bennett et al., 2010) and internal innovation program drivers. The intent would be to deploy within three weeks of team formation, with the purpose of giving team and program leads advanced notice of potential fault lines (Bezrukova, 2013; Lencioni, 2002), and also at the conclusion of the project to allow members and leaders to reflect on performance and deploy best practice to future teams they were part of.

Although no definitive correlations between CQS scores and cultural origins of members was evident, the more culturally diverse teams generally scored highly in most components. The major variant was again socio-linguistic ability. All participants assigned high value to the assessments, feedback indicating that it had identified blind spots and allowing them to develop appropriate tactics and strategy to improve interactions within the team and in general. Likewise, teams reported significant benefit from Four Sight assessment, allowing members to adopt appropriate means to interact with team members with alternate preferences. Of interest, the most culturally diverse team (by all evaluative measures also one of the most productive and successful) with representatives from a total of 7 cultural clusters recorded $50 \%$ of the group being classified as integrators in the Four Sight assessment (Figure 4, Team I). This is now the subject of internal study but immediately poses the natural question on whether teams recruit like-minded members, or if team diversity promotes more holistic behavior in the group. Irrespective, use of these tools (Team Assessment, CQS, Four Sight and SciTS) and deployment of coaches will now form

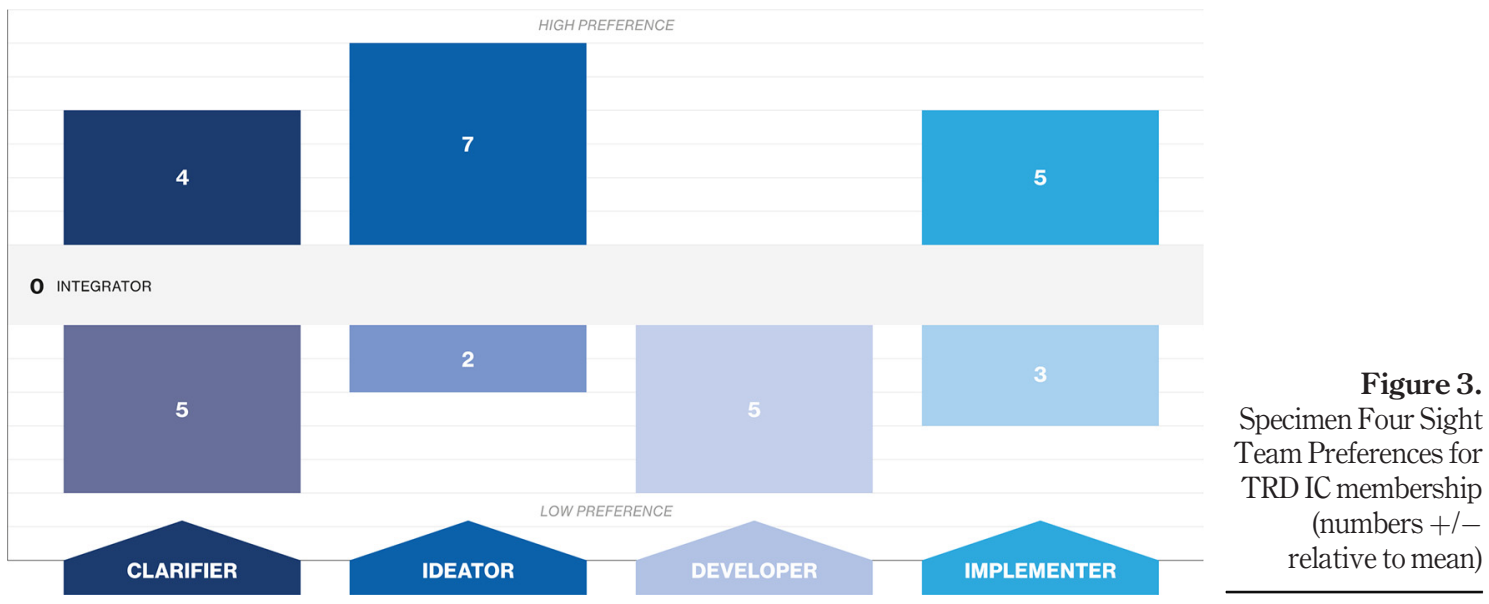


IJIS

13,2

140

an integral component of future rounds of the XFIT innovation program based on these learnings.

Finally, correlation between cultural diversity and innovation capacity of the teams was assessed. Two cluster groups emerged from analysis of the composition, size and outputs of teams (Figure 4). Of the nine teams, two experienced delays requiring time extension (Teams $\mathrm{G}$ and $\mathrm{H}$ ). Teams $\mathrm{E}, \mathrm{F}$ and I completed on time and all generated significant new intellectual property over and above their stated goals. They also represent the more culturally diverse teams. On the other hand, success was also evident for Teams A-D, but in most cases the proof of concepts have been deployed directly to the respective line function activities. These learnings could be classified as implementation oriented components of innovation, complementing the ideation-centric outputs for Teams E-I. An underlying hypothesis could be that smaller (and consequently) less diverse teams are better equipped to implement ideas, while numerically larger teams can more fully exploit diversity for ideation. To test these principles at sufficient power and scale, controls will need to be deliberately introduced and will form the basis of future studies.

\begin{tabular}{|c|c|c|c|c|c|c|}
\hline \multicolumn{7}{|l|}{ \# Team members } \\
\hline \multicolumn{7}{|l|}{ \# Cultural Clusters } \\
\hline \# Time zones from hub & $+\# \mathrm{~h}$ & $-\quad \# \mathrm{~h}$ & & & & \\
\hline Sub-team formation & no $\square$ & yes $\square$ & \# & & & \\
\hline Roadblocks \& barriers & no & yes & \# & & & \\
\hline \multicolumn{7}{|l|}{ \# New ideas to team } \\
\hline \multicolumn{7}{|l|}{ \# Curated ideas to PoC } \\
\hline \multicolumn{7}{|l|}{ \# PoC to implemented } \\
\hline & & & & & & \\
\hline & & poor & marginal & satisfactory & good & excellent \\
\hline \multicolumn{7}{|l|}{ Progress against goals } \\
\hline \multicolumn{7}{|l|}{ Availability of resources } \\
\hline \multicolumn{7}{|l|}{ Meeting frequency } \\
\hline \multicolumn{7}{|l|}{ Meeting format } \\
\hline \multicolumn{7}{|l|}{$\begin{array}{l}\text { Team member } \\
\text { engagement level }\end{array}$} \\
\hline \multicolumn{7}{|l|}{ Team member attitudes } \\
\hline \multicolumn{7}{|l|}{ Keeping to schedule } \\
\hline \multicolumn{7}{|l|}{ Trust levels } \\
\hline \multicolumn{7}{|l|}{ Openness levels } \\
\hline \multicolumn{7}{|l|}{$\begin{array}{l}\text { Responsiveness to } \\
\text { concerns }\end{array}$} \\
\hline \multicolumn{7}{|l|}{$\begin{array}{l}\text { Dispute resolution } \\
\text { methods }\end{array}$} \\
\hline 5 Fault lines & & Absenc & Fear of conflict & & $\begin{array}{l}\text { Accountability } \\
\text { avoidance }\end{array}$ & Inatter \\
\hline
\end{tabular}

Figure 4.

Team performance and inclusivity tracking tool

\section{introdiced and will form the bas of future studies.}




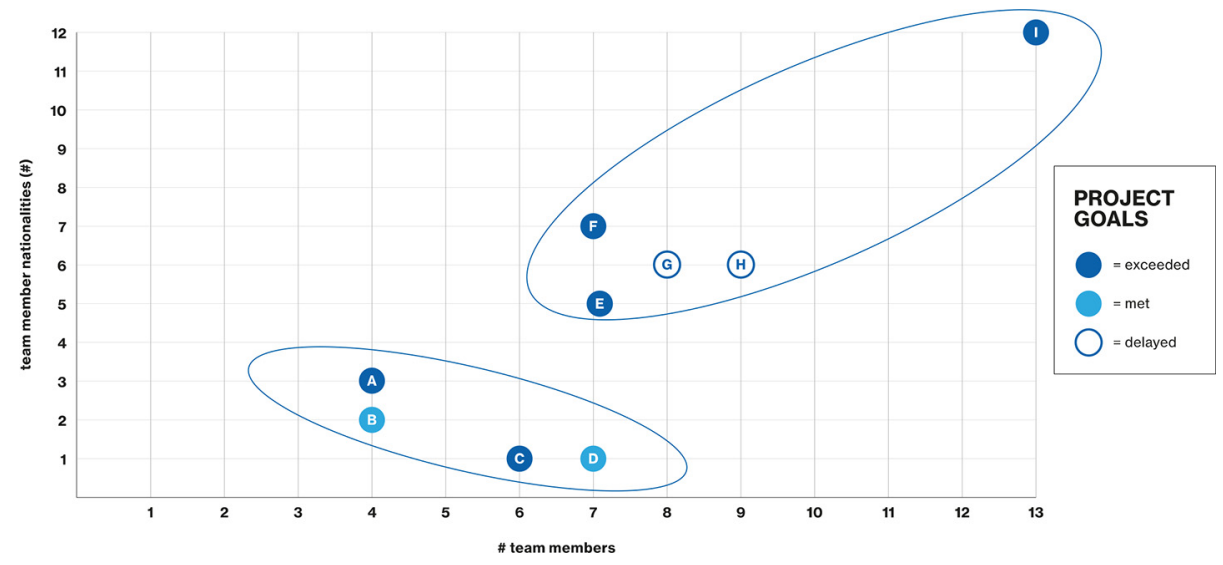

Cultural diversity drives innovation

\section{Conclusions}

There is growing evidence that cultural diversity in teams has a positive impact on innovation performance. This said, managing highly creative culturally diverse teams requires careful attention to team dynamics and promotion of inclusive behaviors to capitalize on the teams' full potential. Cultural assessment instruments, thinking profile analysis and SciTS-inspired coaching can play a positive role in team development and performance by driving behaviors which prevent formation of fault lines. Based on a study of nine innovation teams, evidence supports team diversity having a beneficial impact on both the ideation and implementation phases of innovation. The following specific recommendations resulted from the study:

- Appointing a dedicated meeting "observer" is recommended for medium-large teams to drive inclusion, alerting the meeting chair to relevant touch points in real time.

- Providing opportunities for sub-groups to pre-socialize ideas to drive consensus prior to public presentation and endorsement, minimizing potential for ad hominem interactions.

- Deploying electronic tools to democratize member inputs and questions in meetings.

- Increasing sensitivity to meeting etiquette to prevent potential culturally related biases.

- Deploying team performance tracking tools to help leaders assess inclusivity in real time.

- Development of internal tools which emulate CQS and Four Sight assessments, and SciTS learnings for deployment in ideation projects involving large teams.

\section{Implications}

The majority of innovation projects conducted in the pharmaceutical industry are carried out in teams which range in size, location and composition. To maximize the creative advantages of team diversity, it is necessary to operate in fully inclusive environments. In the case of culturally diverse teams, refined intra-team dynamics and cross-cultural competence $(\mathrm{CQ})$ among team members correlates with superior performance. Accordingly, it is desirable for teams to consider individual and group assessment methodologies prior to 
or soon after the project initiation phase to reduce the potential for fault-lines derived from culturally related mis-understandings and mis-interpretations. Team performance tracking should include monitoring of its operating environment to ensure inclusivity is being maximized. The implications of such tactics for innovation teams across the industry will evolve over time. It is also relevant to consider the context of innovation itself within large and complex organizations. As articulated by Nelson, for innovation teams there may be differences in perspectives and motivation between the organization and the teams and individuals conducting the activities (Nelson, 1991). In this respect, the nine teams in the present study were conducting innovation projects on topics novel to the business, but with the intent of incorporating findings to existing areas of business interest. While this may not represent the classic embodiment of white space innovation in terms of developing new business models (reflecting the fact that TRD is primarily focused on development and implementation of ideas generated by the discovery teams), the potential for new intellectual property was evident for each project. Accordingly, we believe the findings are of relevance to innovation teams across the pharmaceutical and adjacent chemistry intensive industries.

\section{Future research}

Based on the findings herein, it will be appropriate to track team performance over extended periods and multiple projects in an effort to increase learnings on cultural dynamics and their role in the innovation process. It will also be insightful to study the dynamics of team formation itself and whether selection criteria applied have an influence on performance, e.g. by comparing selfassembled teams and deliberately assembled teams. In concert with these actions, considerable emphasis will also be placed on studying team dynamics under virtual/telecom-based operational models. This will become increasingly important for global, dispersed innovation teams as corporate responsibilities will likely limit future long distance air travel based on environmental footprints. Similarly, the unfamiliar working conditions mandated by the COVID-19 global pandemic emphasizes the need for innovation team models that work effectively at scale and over extended time periods under remote conditions. The global pharmaceutical industry is ideally positioned to benefit from these studies and observations and we hope the findings will stimulate impact studies across the sector. Additionally, the principles should also be applicable to adjacent industries where innovation (ideation and implementation phases) is key, team dynamics are complex, and cultural and geographic diversity is high (Mayer and Bello, 2012). It will also be desirable for industries to collectively generate and disseminate aggregate data on the ties between cultural diversity and innovation performance at scale in order to underscore the economic impact. Such can help identify future research topics at the interfaces of social science and corporate innovation (Farooq, 2017).

\section{References}

Amiri, A.N., Moghimi, S.M. and Kazemi, M. (2010), "Studying the relationship between cultural intelligence and employees' performance", European Journal of Scientific Research, Vol. 42, pp. $432-441$.

Belbin, M. (2010), Roles at Work, 2nd ed., Imprint Routledge, London, doi: 10.4324/9780080963242.

Bennett, L.M. Gadlin, H. and Levine-Finley, S. (2010), "Collaboration and team science: a field guide", available at: www.cancer.gov/about-nci/organization/crs/research-initiatives/team-science-fieldguide/collaboration-team-science-guide.pdf (accessed 10 April 2020).

Bertelsmann (2018), "Cultural diversity has a positive impact on innovation", available at: www. bertelsmann-stiftung.de/en/our-projects/living-diversity/project-news/cultural-diversity-has-apositive-impact-on-innovation/ (accessed 10 April 2020). 
Bezrukova, K. (2013), "Understanding and addressing faultlines", available at: http:/sites.nationalacademies. org/cs/groups/dbassesite/documents/webpage/dbasse_083763.pdf (accessed 10 April 2020).

Bratsberg, H.M. (2012), "Empathy maps of the four sight preferences", available at: https:/pdfs. semanticscholar.org/d4e9/e13a43847ce1ecebec806206f2d8fe676dad.pdf (accessed 10 April 2020).

Dakhli, M. and de Clercq, D. (2004), "Human capital, social capital, and innovation: a multi-country study", Entrepreneurship and Regional Development, Vol. 16 No. 2, pp. 107-128.

De Abreu Dos Reis, C.R., Sastre Castillo, M.Á. and Roig Dobón, S. (2007), "Diversity and business performance: 50 years of research", Service Business, Vol. 1 No. 4, p. 257.

Farooq, R. (2017), "A framework for identifying research gap in social sciences: evidence from the past", The IUP Journal of Management Research, Vol. 16 No. 4, pp. 66-75.

Gibson, C.B. and Gibbs, J.L. (2006), "Unpacking the concept of virtuality: the effects of geographic dispersion, electronic dependence, dynamic structure, and national diversity on team innovation", Administrative Science Quarterly, Vol. 51 No. 3, pp. 451-495.

Gudykunst, W.B. and Ting-Toomey, S. (1988), Culture and Interpersonal Communication, SAGE Publications, ISBN: 0803929455.

Hampden-Turner, C. and Trompenaars, F. (2006), "Cultural intelligence: is such a capacity credible?", Group and Organization Management, Vol. 31 No. 1, pp. 56-63.

Higgs, M. (1996), "Overcoming the problems of cultural differences to establish success for international management teams", Team Performance Management: An International Journal, Vol. 2 No. 1, pp. 36-43.

Hofstede, G. (2010), "The GLOBE debate: back to relevance", Journal of International Business Studies, Vol. 41 No. 8, pp. 1339-1346.

Horwitz, S.K. and Horwitz, I.W. (2007), "The effects of team diversity on team outcomes: a metaanalytic review of team demography", Journal of Management, Vol. 33 No. 6, pp. 987-1015.

Jackson, S.E., May, K.E. and Whitney, K. (1995), "Understanding the dynamics of diversity in decisionmaking teams", in Guzzo, R.A. and Salas, E. (Eds), Team Decision-Making Effectiveness in Organizations, Jossey-Bass, San Francisco, pp. 204-261.

Jones, G.B., Chirino-Chace, B. and Wright, J.M. (2020), "Cultural diversity drives innovation: empowering teams for success", Int. J. Innov. Sci, pending, Vol. 12.

Kaasa, A. and Vadi, M. (2010), "How does culture contribute to innovation? Evidence from European countries", Economics of Innovation and New Technology, Vol. 19 No. 7, pp. 583-604.

Kivunja, C. (2015), "Using De Bono's six thinking hats model to teach critical thinking and problem solving skills essential for success in the 21st century economy", Creative Education, Vol. 6 No. 3, pp. 380-391.

Koehne, G., Shih, P.C. and Olson, J.S. (2012), "Remote and alone: coping with being the remote member on the team", Proceedings of the ACM Conference on Computer Supported Cooperative Work, ACM, New York, NY, pp. 1257-1266.

Lau, D.C. and Murnighan, J.K. (1998), "Demographic diversity and faultlines: the compositional dynamics of organizational groups", Academy of Management Review, Vol. 23 No. 2, p. 325.

Lencioni, P. (2002), The Five Dysfunctions of a Team, Jossey-Bass, San Francisco.

Lorenzo, R. Voigt, N. Tsusaka, M. Krentz, M. and Abouzahr, K. (2018), "How diverse leadership teams boost innovation”, Boston Consulting Group, available at: www.bcg.com/publications/2018/ how-diverse-leadership-teams-boost-innovation.aspx (accessed 10 April 2020).

Matsumoto, D. and Hwang, H.C. (2013), "Assessing cross-cultural competence: a review of available tests", Journal of Cross-Cultural Psychology, Vol. 44 No. 6, pp. 849-873.

Mayer, M. and Bello, Y. (2012), "Leading cross cultural teams in today's global marketplace”, available at: www.pmi.org/learning/library/cross-cultural-teams-global-marketplace-6304 (accessed 10 April 2020).

Moon, T. (2013), "The effect of cultural intelligence on performance in multicultural teams", Journal of Applied Social Psychology, Vol. 43 No. 12, pp. 2141-2425. 
Nakata, C. and Sivakumar, K. (1996), "National culture and new product development: an integrative review", J. Prod. Innov. Manag, Vol. 13, pp. 462-463.

Nelson, R.R. (1991), "Why do firms differ, and how does it matter?", Strategic Management Journal, Vol. 12, pp. 61-74.

Novartis (2020), available at: www.fastcompany.com/company/novartis

Shane, S. (1993), "Cultural influences on national rates of innovation", Journal of Business Venturing, Vol. 8 No.1, pp. 59-73.

SHRM (2015), "Cultural intelligence: the essential intelligence for the 21st century”, available at: https:// culturalq.com/wp-content/uploads/2016/05/SHRM-report.pdf (accessed 10 April 2020).

Thomas, K.W. and Kilmann, R.H. (1978), "Comparison of four instruments measuring conflict behavior", Psychological Reports, Vol. 42 No. 3_suppl, pp. 1139-1145.

Van Wulfen, G. (2009), “The perfect innovation team”, available at: https://innovationmanagement.se/ 2013/07/10/the-perfect-innovation-team/ (accessed 10 April 2020).

Viki, T. (2016), "Eight key personality types for innovation teams", available at: www.forbes.com/sites/ tendayiviki/2016/10/11/the-eight-personalities-you-need-in-your-innovation-team/\#2cf9ee9c3320 (accessed 10 April 2020).

\section{Corresponding author}

Graham Jones can be contacted at: graham.jones@novartis.com

For instructions on how to order reprints of this article, please visit our website: 